\title{
Relation between synovial fluid C3 degradation products and local joint inflammation in rheumatoide arthritis, osteoarthritis, and crystal associated arthropathy
}

\author{
MICHAEL DOHERTY, ${ }^{1}$ NORA RICHARDS, ${ }^{2}$ JUNE HORNBY, ${ }^{1}$ AND \\ RICHARD POWELL ${ }^{2}$
}

From the ${ }^{1}$ Rheumatology Unit, City Hospital, Nottingham; and the ${ }^{2}$ Department of Immunology, Queen'⿺ Medical Centre, Nottingham

SUMMARY C3 degradation products (C3dg/d) were estimated in 288 synovial fluid (SF) samples $\stackrel{\text { ? }}{3}$ (rheumatoid arthritis (RA) 93, osteoarthritis (OA) 68, chronic pyrophosphate arthropathy 80 acute pseudogout 20, others 27) from knees of 138 patients (bilateral 67, serial sampling on two tक्ठ six occasions 40). At each aspiration knees were defined as 'active' or 'inactive' by single observe? global assessment using six clinical parameters of inflammation. Lack of correlation betweerso paired SF and plasma C3dg/d implied local C3 activation within joints. Raised SF C3d levels were found in active compared with inactive RA joints (mean (range) 51 (15-105) and 6 (0-158 units $/ \mathrm{ml}$ respectively). Low SF C3dg/d levels were found in OA (mean (range) $0.8(0-7) \mathrm{units} / \mathrm{ml}$ and chronic pyrophosphate arthropathy (mean (range) $4(0-16) \mathrm{units} / \mathrm{ml}$ ), irrespective of clinica activity. In contrast, very high levels (mean (range) 61 (16-126) units $/ \mathrm{ml}$ ) were present in all case of pseudogout. These differences remained after correction for SF C3 or albumin. This study is the first to show a positive correlation between SF C3dg/d and local inflammation in RA joints. I $\overrightarrow{\mathrm{P}}$ further suggests that $\mathrm{C} 3$ activation is a constant feature of pseudogout but not an accompaniment of inflammation associated with chronic crystal associated synovitis or OA.

Key words: complement, C3dg, C3d, clinical activity, crystal synovitis, pyrophosphatẹ़े arthropathy.

Complement activation by both classical and alternative pathways is thought to play an important part in mediation of inflammation in a variety of rheumatic diseases. ${ }^{1-7}$ Evidence to support such a role includes $(a)$ in vitro activation of complement by factors such as immune complexes, ${ }^{8}$ rheumatoid factors, ${ }^{910}$ and monosodium urate and calcium pyrophosphate dihydrate (CPPD) crystals $^{11-13}$ and (b) direct demonstration of complement activation in blood and synovial fluid (SF) of patients with rheumatic disease ${ }^{1-7}$

Although complement activation in rheumatic diseases has been extensively investigated in plasma, there are relatively few substantial

Accepted for publication 2 September 1987.

Correspondence to Dr Michael Doherty, Rheumatology Unit, City Hospital, Nottingham NG5 1PB. studies of in vivo complement activation within joints. ${ }^{1-6}$ 14-26 Such studies, however, using क् variety of assay systems, have demonstrated $S$ complement activation particularly in rheumatoi arthritis, ${ }^{1-6} 14-25$ gout, 1614161824 pseudogout 614151824 systemic lupus erythematosus, ${ }^{6} 10$ and seronegative spondarthropathies, ${ }^{161516}$ buf not in osteoarthritis. ${ }^{13-616182021} 24$ Marked variability within each disease group and consider able overlap between diagnostic categories have uniformly been reported. ${ }^{3-6} 14-25$ Only two studies however, have attempted to characterise the clinicaff activity of the joints aspirated, and in these reports, including 40 patients with rheumatoid arthritis ${ }^{21}$ an $\$$ 10 with juvenile chronic arthritis, ${ }^{26}$ no apparen correlation was observed between SF complemeng activation and the clinical state of the joint. The relation between complement activation and 
local joint inflammation, therefore, remains in question. $^{21} 24$

In this study we estimated complement degradation products with $\mathrm{C} 3 \mathrm{~d}$ specificity $\left(\mathrm{C} 3 \mathrm{dg} / \mathrm{d}^{27}\right.$ hereafter abbreviated as $\mathrm{C} 3 \mathrm{~d}$ ) together with $\mathrm{C} 3$ in paired SF and plasma samples from a large number of patients with well characterised joint disease. For comparative purposes all SF aspirations were from a single site (the knee). Unlike most previous studies we assessed the clinical state of each joint aspirated, performed frequent bilateral sampling (often from knees showing different inflammatory activities), and used serial sampling of the same knee during differing phases of clinical activity.

\section{Patients and methods}

Approval for this study was obtained from the local ethical committee.

\section{S U B JECTS}

One hundred and thirty eight patients attending the rheumatology unit of the City Hospital with clinically evident disease affecting at least one knee were included in the study. Minimum investigation in each case included full blood count, erythrocyte sedimentation rate, serum rheumatoid factor, plain radiographs of hands, feet, and knees, and examination of fresh knee SF for birefringent crystals (compensated polarised light microscopy); further investigation was determined by the individual patient characteristics. The principal diagnostic categories were rheumatoid arthritis (RA), osteoarthritis (OA), and crystal associated arthropathy. All patients with RA had erosive disease and fulfilled American Rheumatism Association criteria for classic or definite disease ${ }^{28}$; patients designated as seropositive had raised titres of serum $\mathrm{IgG}$ rheumatoid factor $^{29}$ recorded on at least one occasion. Patients with OA had symptomatic seronegative gonarthrosis with knee radiographs showing cartilage loss plus subchondral sclerosis or osteophyte, or both; none had radiographic chondrocalcinosis, SF CPPD crystals, or evidence of other primary joint disease. Patients with pseudogout had typical self limiting acute episodes of knee synovitis with SF CPPD crystals and no evidence of coexistent joint disease (e.g., sepsis). Chronic pyrophosphate arthropathy (CPA) was defined as persistent ( $>3$ months) symptomatic gonarthrosis with SF CPPD crystals and $x$ ray features of OA (often with predominant patellofemoral involvement, bi- or tricompartmental disease, or chondrocalcinosis). Gout was confirmed by demonstration

Table 1 Diagnosis and details of patients

\begin{tabular}{|c|c|c|c|c|c|}
\hline Diagnosis & No & $F: M$ & $\begin{array}{l}\text { Age mean } \\
\text { (range) (years) }\end{array}$ & $\begin{array}{l}\text { Duration of } \\
\text { knee symptoms } \\
\text { mean (range) }\end{array}$ & $\begin{array}{l}\text { SF } \\
\text { samples }\end{array}$ \\
\hline \multicolumn{6}{|l|}{$\mathbf{R A}$} \\
\hline Seropositive & 33 & $22: 11$ & $63(42-82)$ & $8(1-34)$ yr & 74 \\
\hline Seronegative & 6 & $2: 4$ & $62(45-76)$ & $4(1-14)$ yr & 19 \\
\hline Total & 39 & $24: 15$ & $63(42-82)$ & $7(1-34)$ yr & 93 \\
\hline OA & 36 & 19:17 & $67(45-89)$ & $6(1-25)$ yr & 68 \\
\hline CPA & 33 & $19: 14$ & $72(31-88)$ & $8(1-31)$ yr & 80 \\
\hline Pseudogout & 10 & $8: 2$ & $74(53-94)$ & $12-72 \mathrm{~h}$ & 20 \\
\hline Chronic gout & 5 & $1: 4$ & $63(54-77)$ & $6(2-51) y r$ & 5 \\
\hline Acute gout & 3 & $0: 3$ & $43(35-50)$ & $12-72 \mathrm{~h}$ & 3 \\
\hline Psoriatic arthritis & 5 & $2: 3$ & $32(18-57)$ & $1(0.5-2)$ yr & 8 \\
\hline Acute Reiter's disease/reactive & 7 & $3: 4$ & $31(20-62)$ & $0.8(0.1-2)$ yr & 11 \\
\hline
\end{tabular}

Table 2 Clinical assessment of knees

\begin{tabular}{lll}
\hline & Inactive & Active \\
\hline Individual parameters & & Present \\
Increased warmth & Absent & Moderate-marked/tense \\
Effusion & Absent-mild/not tense & Present \\
Synovial thickening & Absent & $2-3$ \\
Joint line tenderness (0-3) & $0-1$ & 1 hour or more \\
Early morning stiffness & $<1$ hour & 15 minutes or more \\
Inactivity stiffness & $<15$ minutes & $4-6$ parameters active \\
Global assessment & $<3$ parameters active & 4 \\
\hline
\end{tabular}


of monosodium urate crystals in fresh knee SF. The diagnosis of other conditions was similarly based on clinical presentation/distribution of arthropathy together with appropriate serological, radiographic, and SF characteristics; no patient with indeterminate or 'probable' disease was included. Patient details are shown in Table 1.

\section{METHODS}

Two hundred and eighty eight knee aspirations were performed on these 138 patients (bilateral on the same occasion 58, serial sampling of the same knee on two to six occasions 40). At each aspiration knees were examined and assessed by a single observer for six clinical parameters of inflammation-namely, increased warmth, effusion (0-3), anterior joint line and suprapatellar synovial thickening, joint line tenderness (0-3), early morning stiffness, and inactivity stiffness (Table 2). The joint was globally assessed as 'active' if four to six parameters were active, and 'inactive' if less than three parameters were active; 23 additional knees scoring three active parameters, i.e., 'intermediate', were not included. For complement estimations paired blood and SF samples were taken immediately into edetic acid (10 $\mathrm{mmol} / \mathrm{l}$ ), samples were then centrifuged for five minutes, and the plasma and SF stored in vapour phase liquid nitrogen $\left(-186^{\circ} \mathrm{C}\right)$ within three hours of collection.

C3d levels (units $/ \mathrm{ml}$ ) were measured by the double decker rocket immunoelectrophoresis method of Brandslund et al. ${ }^{27}$ Essentially, $4 \mu$ l edetic acid plasma were electrophoresed overnight $(2.5$ $\mathrm{V} / \mathrm{cm}$ ), initially through a gel layer containing antihuman C3c (Dako) and then through a layer containing antihuman C3d (Dako) where the C3d rockets were formed. After drying and staining, the rocket heights of test samples and standards were measured and the $\mathrm{C} 3 \mathrm{~d}$ values calculated from a standard curve. The $\mathrm{C} 3 \mathrm{~d}$ content of the standard (normal serum incubated at $37^{\circ} \mathrm{C}$ for four days) had previously been assigned an arbitrary value of 100 units $/ \mathrm{ml}$, and the upper limit of normal for plasma was statistically determined to be 12 units $/ \mathrm{ml}$. There is no normal range for SF, very low SF levels $(<5$ units/ml) cannot be accurately estimated by this method and were counted as 0 for statistical analysis. Plasma and SF C3 levels $(\mathrm{g} / \mathrm{l})$ and SF albumin $(\mathrm{g} / \mathrm{l})$ were estimated by a turbidometric method on a centrifugal fast analyser. ${ }^{30}$

Correlation between plasma and SF levels was determined by Deming's procedure. Comparison of SF findings between active and inactive joints and between different diagnostic categories was by the Wilcoxon test when the number of values did not exceed 25 in the smaller group and 50 in the larger 
group, and by Student's $t$ test when any group contained larger numbers than this.

\section{Results}

COMPARISON BETWEEN PLASMAANDSFC 3 d LEVELS

Paired plasma and SF findings in the different disease categories are shown in Table 3; the lower number of plasma samples (221) relates to frequent bilateral knee sampling on the same occasion.

Comparison between groups showed raised plasma C3d levels only in seropositive RA patients $(\mathrm{p}<0.01)$. Correction for $\mathrm{C} 3$ (i.e., the plasma $\mathrm{C} 3 \mathrm{~d} / \mathrm{C} 3$ quotient ${ }^{4}{ }^{31}$ ) more clearly delineated the raised level found in this group (Table 3 ).

Comparison between major groups for SF C3d showed markedly raised levels in seropositive RA $(p<0.001)$, seronegative RA $(p<0.001)$, and pseudogout $(\mathrm{p}<0.001)$ compared with the very low levels in OA (Table 3); a modest, but highly significant, increase was seen in CPA $(p<0.001)$. The highest levels were seen in pseudogout (mean (range) 61 (16-126) units $/ \mathrm{ml}$ ). In the other, smaller groups a moderate increase of SF C3d was seen in acute Reiter's disease $(p<0 \cdot 01)$, psoriatic arthritis $(\mathrm{p}<0.01)$, and in the three patients with acute gout; levels in chronic gout were similar to those in CPA. Correction for SF C3 did not significantly alter these between group comparisons but emphasised the increases seen in seropositive RA, pseudogout, and acute gout (Table 3). Correction for SF albumin similarly did not alter the differences between groups but emphasised the increases seen in pseudogout and seropositive RA (Table 3).

No correlation was observed between plasma and SF C3d levels either overall $(r=0.44)$ or within the seropositive RA group alone $(r=0 \cdot 40)$. The higher range of $C 3 d$ and $C 3 d / C 3$ values for $S F(0-126$, mean (SD) $19.1(27.6)$ units $/ \mathrm{ml}$ and 0-221, mean
(SD) $28.8 \quad(45.4)$ respectively) compared with plasma (0-28, mean (SD) $11.6(6.0)$ units/ml and 0-63, mean (SD) $9 \cdot 5(5 \cdot 7)$ respectively) supported local activation of $\mathrm{C} 3$ within joints.

\section{CORRELATION BETWEEN SF C3d AND}

\section{CLINICAL ACTIVITY}

Mean SF C3d, C3d/C3, and C3d/albumin values in different diagnostic groups divided according to clinical joint activity are shown in Table 4; comparable data for groups requiring no division (i.e., pseudogout, acute gout, Reiter's disease-äll joints active; chronic gout-all joints inactive) are contained in Table 3. Individual SF C3d values are shown in Fig. 1.

Markedly raised SF C3d levels were found in active compared with inactive RA joints, irrespective of seropositivity ( $p<0.001$ both groups). No difference in SF C3d, however, was observed between active or inactive OA, or between active and inactive CPA; compared with active OA, SF C3d remained significantly raised in both active $(\mathrm{p}<0.001)$ and inactive $(\mathrm{p}<0.001)$ CPA knees. In contrast with active CPA, markedly raised SF C3d levels were seen in all cases of pseudogout $(p<0 \cdot 001)$.

Compared with pyrophosphate arthropathy, a less impressive difference in SF C3d was observed between the small number of patients with acute (active) and chronic (inactive) gout (Fig. 1). SF C3d levels in active psoriatic arthropathy were comparable with those in acute Reiter's disease (Fig. 1).

SF C 3 d IN BILATERAL AND SERIAL SAMPLES Twelve patients with RA underwent simultaneous bilateral sampling at a time when their knees showed contrasting clinical activities. Comparison between the two sides in these patients showed marked differences in SF C3d, with higher levels in the active knee (Fig. 2). Simultaneous bilateral sampling of 18 patients with RA and clinically

Table 4 SF C3d findings in different diagnostic groups divided according to clinical activity of the aspirated joint ${ }^{*}$

\begin{tabular}{|c|c|c|c|c|c|}
\hline & & Number & C3d (units/ml) & $C 3 d / C 3$ & C3d/albumin \\
\hline \multicolumn{6}{|l|}{ RA } \\
\hline \multirow[t]{2}{*}{ Seropositive: } & Active & 57 & $51 \cdot 2(24)$ & $96.4(48)$ & $2 \cdot 27(1.45)$ \\
\hline & Inactive & 17 & $3.8(4.8)$ & $10.8(17)$ & $0.21(0.32)$ \\
\hline \multirow[t]{2}{*}{ Seronegative: } & Active & 9 & $49 \cdot 1(30)$ & $39 \cdot 3(28)$ & $2.82(1.09)$ \\
\hline & Inactive & 10 & $0.8(2 \cdot 2)$ & $1.0(3.2)$ & $0.04(0.10)$ \\
\hline \multirow[t]{2}{*}{$\mathbf{O A}$} & Active & 31 & $0.9(2 \cdot 1)$ & $1.0(2.7)$ & $0.04(0.08)$ \\
\hline & Inactive & 37 & $0.6(1.7)$ & $1.5(3.7)$ & $0.23(0.09)$ \\
\hline \multirow[t]{2}{*}{ CPA } & Active & 27 & $4.5(5 \cdot 2)$ & $6.8(10.8)$ & $0.22(0.36)$ \\
\hline & Inactive & 53 & $3.5(4 \cdot 2)$ & $4.2(9.8)$ & $0.14(0.21)$ \\
\hline \multirow[t]{2}{*}{ Psoriasis } & Active & 6 & $14 \cdot 3(4 \cdot 1)$ & $21 \cdot 4(12)$ & $0.95(1 \cdot 11)$ \\
\hline & Inactive & 2 & $0(0)$ & $0(0)$ & $0(0)$ \\
\hline
\end{tabular}

${ }^{*}$ Results are mean (SD). 


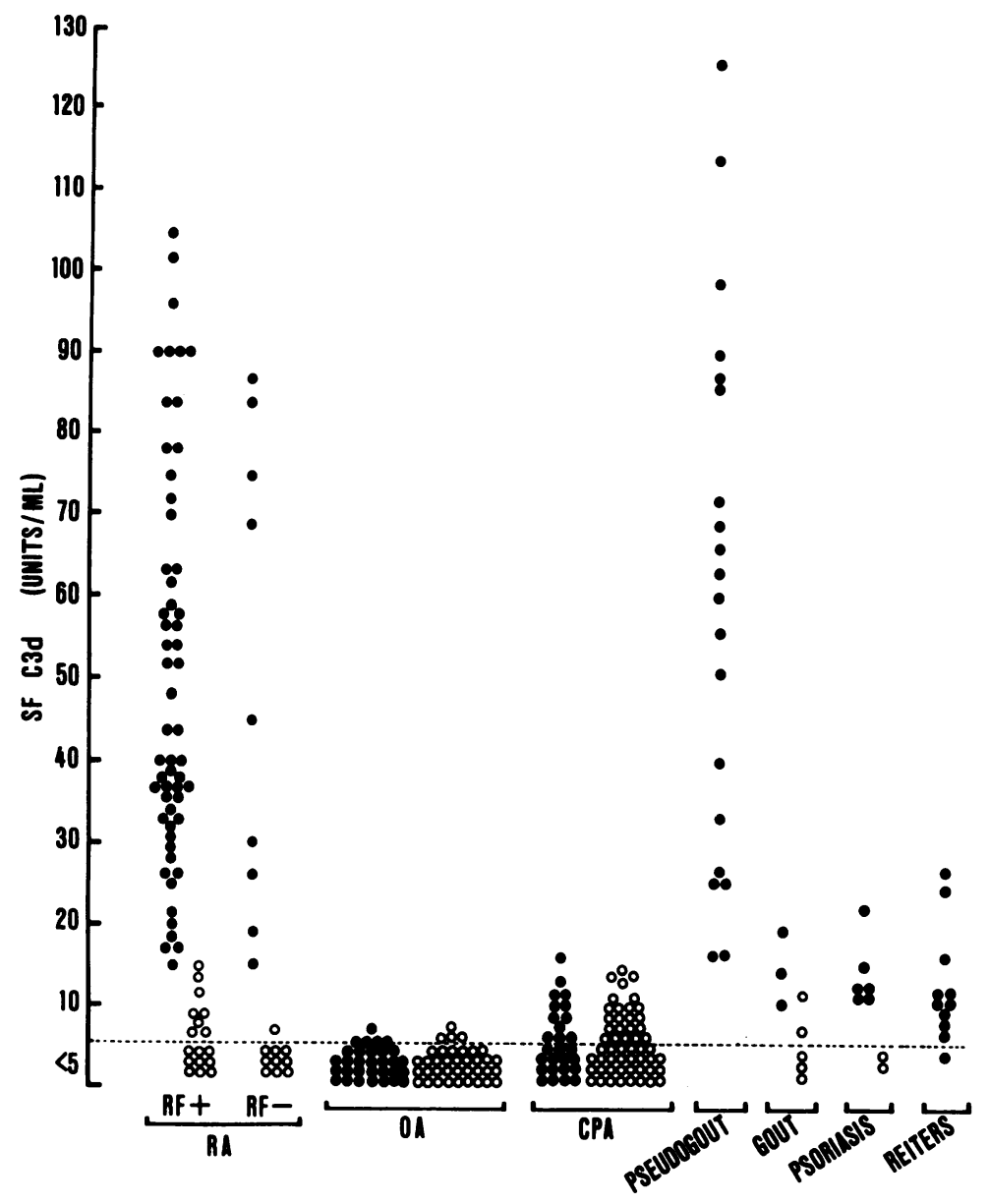

Fig. 1 Synovial fluid C3d levels in active (O) and inactive (O) knees. ( $R F=$ rheumatoid factor.)

similar knees (Fig. 2) also showed frequent marked differences in SF C3d between the two active joints in the same individual (mean (SD) difference $19.7(21.4)$, range $1-60$ units/ml; $n=15)$. For the three patients with bilateral inactive knees all values were $<5$ units $/ \mathrm{ml}$. Owing to the narrow range of SF C3d values, simultaneous bilateral sampling in patients with OA and CPA showed only minor differences between joints of the same individual, irrespective of clinical activity. Similar differences to those observed in RA were seen, however, in the few bilateral samples of patients with pseudogout (four), psoriatic arthritis (two), and Reiter's disease (three).

Five patients with.RA underwent serial sampling of the same knee during different phases of clinical activity (aspirations were performed at varying intervals over a 12 month period). Marked changes in SF C3d were observed, with raised levels occur ring at times of clinical activity (Fig. 3). As with? bilateral aspiration, serial sampling of $O A$ and $C P A D$ knees showed only minor differences in SF C3d을 irrespective of clinical activity.

\section{Discussion}

Reduction in individual native complement compo:0 nents is an unreliable indicator of complemento activation in vivo. Enhanced hepatic synthesis of complement factors as acute phase reactants ${ }^{32}$ and? variable synthesis by extrahepatic tissue, including 0 synovium, ${ }^{33}$ may permit significant activation of $\mathrm{C} 3$. without concomitant decrease in $\mathrm{C} 3$ concentration? outside the normal range. ${ }^{34}$ Conversely, low $C 3 \stackrel{\circ}{\Omega}$ levels may reflect decreased synthesis rather than $\mathrm{C}_{30}$ consumption. ${ }^{35}$ Evaluation of in vivo complement $\mathrm{C}$ 

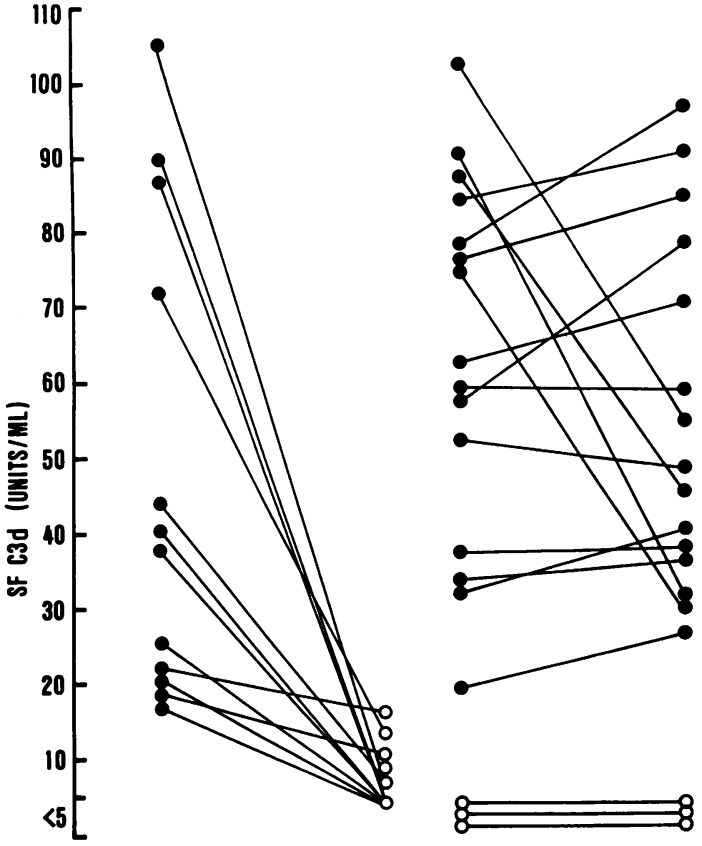

Fig. 2 Synovial fluid C3d levels in patients with $R A$ who underwent bilateral aspiration of knees showing differing (left) or similar (right) clinical inflammatory activity. $\mathrm{O}=$ active; $\mathrm{O}=$ inactive.

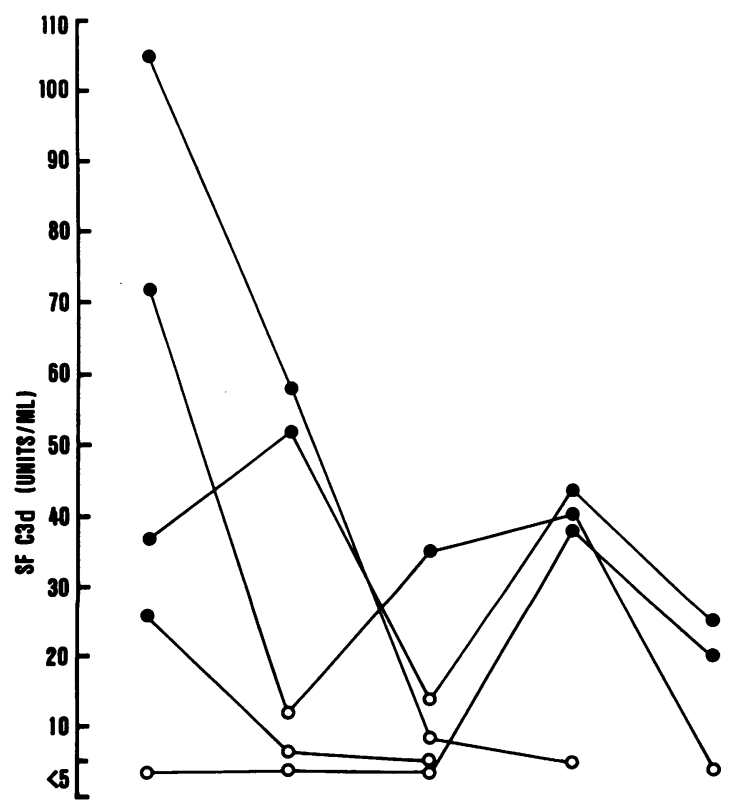

Fig. 3 Serial synovial fluid C3d levels in five patients with $R A$ who showed varying clinical activity on different occasions during a 12 month period. $\mathrm{O}=$ active; $\bigcirc=$ inactive. activation is therefore ideally based on detection of activation products or on turnover studies. In the present study evidence of complement activation, by either classical or alternative pathway, was sought by estimation of stable breakdown products of $\mathrm{C} 3$ with $\mathrm{C} 3 \mathrm{~d}$ specificity $(\mathrm{C} 3 \mathrm{dg} / \mathrm{d})$ using an established rocket immunoelectrophoresis system. ${ }^{27}$ As the $\mathrm{C} 3 \mathrm{~d} / \mathrm{C} 3$ ratio may be a more reliable indicator of complement activation than $\mathrm{C} 3 \mathrm{~d}$ alone, ${ }^{431}$ this quotient was additionally determined.

As in previous studies ${ }^{4} 2325$ a marked increase of SF C3d was shown in certain disease categories. That such an increase represents local activation of complement within joints ${ }^{24}{ }^{25}$ was supported by the lack of correlation between plasma and SF C3d, the markedly higher C3d values observed in SF, and the frequently marked variation in SF C3d levels between different joints of the same individual. As the concentration of serum proteins in SF varies considerably the albumin concentration was used to correct absolute $\mathrm{C} 3 \mathrm{~d}$ to relative values, thereby permitting comparison of SF results between different groups. It was not necessary, however, to correct between different groups. It was also unnecessary to correct for protein content in order to show local complement activation in SF (owing to the frequently much higher values in SF than in plasma), and correction for SF albumin made little difference to intergroup or within group comparisons. Similarly, because no group showed marked depression of SF $\mathrm{C} 3$, comparison of the corrected $\mathrm{C} 3 \mathrm{~d} / \mathrm{C} 3$ quotient, ${ }^{431}$ though to some extent sharpening division between categories, did not substantially alter findings derived from comparison of SF C3d alone.

The results of the between group comparisons are in substantial agreement with previous SF complement studies in showing no evidence for complement activation in OA, ${ }^{13-6} 1618202124$ but significant activation in $\mathrm{RA}^{1-614-25}$ (particularly those with seropositive disease, 13615 ) and acute pseudogout. 614151824 This is the first study to examine CPA as a separate major diagnostic group, and the finding of a modest but significant increase of SF C3d in CPA compared with OA has not previously been reported. SF complement activation has previously been demonstrated in psoriatic arthropathy 15162125 and acute Reiter's disease, ${ }^{1} 6162125$ and the only unexpected finding was the comparatively modest increase of SF C3d in the three cases of acute gout. ${ }^{614161824}$ Although possibly explained by the small sample numbers or inappropriate timing of aspiration after onset of the acute attack, this finding is consistent with the suggested minor role for complement activation in acute gouty inflammation. 162136 
The major findings of this study, however, relate to division within each diagnostic group according to the local clinical inflammatory activity of the aspirated joint. There is no generally agreed system for clinical assessment of 'joint inflammation', and any division into active or inactive is necessarily arbitrary and an inevitable compromise. The six clinical parameters employed, however, may reasonably be expected to reflect predominantly inflammatory activity, rather than mechanical damage or periarticular injury, in the disease states examined, and for the purposes of the present study a single observer and a summated score were used with exclusion of joints that were intermediate between the two extremes of the scoring range. Further grading, other than into active or inactive, was not attempted. Such clinical characterisation would seem essential to any study that investigates mediators of inflammation in joint tissues. Surprisingly, however, only two SF complement studies have previously addressed this issue and attempted to define aspirated joints in both clinical and diagnostic terms. Sheppeard et al graded clinical activity of knees as mild, moderate, or severe according to the degree of pain, swelling, warmth, and tenderness, ${ }^{21}$ whereas Mollnes and Paus used a functional knee score (0-100) devised for outcome assessment of RA knees after synovectomy. ${ }^{26}$ Pain, unspecified tenderness, restriction of movement, deformity, and instability, however, do not necessarily reflect the presence of synovitis, and inappropriate clinical assessment may in part explain the failure of either study to show correlation between SF complement activation and clinical activity.

In the present study markedly raised SF C3d levels were seen in active compared with inactive RA joints, and serial sampling showed that SF C3d levels vary in concert with inflammatory activity. To our knowledge this is the first study to show a positive correlation between SF complement activation and local, clinically assessed inflammation in RA joints. A similar correlation was suggested in the two smaller seronegative spondarthritis groups in this study.

Seropositive and seronegative RA patients were considered separately as rheumatoid factors have been shown to activate complement in vitro, ${ }^{910}$ and positive correlation between rheumatoid factor and complement activation has been shown in plasma ${ }^{37}$ and in certain SF studies ${ }^{126}$ of patients with RA. The present finding of a higher mean level of SF C3d in seropositive patients as a group appears to support a possible role for rheumatoid factor in complement activation. ${ }^{1}{ }^{10}$ Comparison of SF C3d in active joints alone, however, as in certain previous reports, ${ }^{52125}$ showed no clear relation between seropositivity and complement activation, thus emphasising the importance of clinical and diagnostic characterisation. The cause(s) or pathways of complement activation in RA, however, were not the subject of the present study and cannot음 be deduced from the data.

Comparison of SF C3d findings in OA, CPA, and pseudogout was of special interest. Although OA joints are commonly used as 'non-inflammatory' disease controls in SF studies, ${ }^{13-616182021}$ many. OA knees in the present study were clinically active $\overrightarrow{\vec{\omega}}$ being associated with marked inflammatory stiffness and showing warm, large effusions, with join $5_{0}$ line/capsular tenderness. Notwithstanding theis presence of obvious clinical inflammation in such joints, no activation of SF C3 could be shown. CPAis a common subset of $\mathrm{OA}$ characterised by $2 \stackrel{\circ}{\circ}$ frequent inflammatory component, atypical distribution, characteristic radiographic features,and the presence of SF CPPD crystals. ${ }^{38}$ Although CPPD crystals may activate complement ${ }^{12} 13$ and other mediators of inflammation in vitro, ${ }^{36} 39 \frac{\mathrm{a}}{\mathrm{P}}$ causal role for these crystals in chronic disease hase been questioned. ${ }^{38}$ Compared with uncomplicatedo $\mathrm{OA}$, the modest but significant increase of SF C3d in active and inactive CPA and the very high levels in pseudogout support a role for CPPD crystals in complement activation in vivo. No significant differ ence in SF C3d was observed, however, between active and inactive CPA, suggesting that, as with $\mathrm{OA}, \mathrm{SF}$ complement activation is not an accomo paniment of the clinical inflammation associated with chronic CPPD crystal associated arthritis These observations may be explained by gross. 'shedding' of naked crystals (with surface character istics capable of activating complement) durings episodes of acute pseudogout, ${ }^{38}{ }^{39}$ but less dramatie release of crystals (with subsequent masking of the active crystal surface by protein coating) during CPA. ${ }^{38-40}$ The role of CPPD crystals in joint disease and the nature of the inflammation associated with $\mathrm{OA}$ and chronic crystal associated synovitis however, remain unresolved.

In the present study considerable additiona information was obtained by further clinical divisions within diagnostic groups. We suggest that future SE studies relating to inflammation or tissue degradao tion take into account the clinical characterisation as well as the diagnosis of joints at time of aspiration. Improved systems of clinical activity assessment must necessarily be agreed and validated

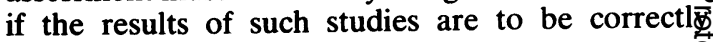
interpreted and compared between centres.

We would like to thank the Arthritis and Rheumatism Council fo financial support, Gillian Hull and April Powell-Richards for 
skilled technical assistance, and Caroline Bloomfield for excellent secretarial work.

\section{References}

1 Zvaifler $\mathrm{N} \mathrm{J}$. Breakdown of $\mathrm{C} 3$ in human synovial fluid. J Clin Invest 1969; 48: 1532-42.

2 Hedberg H, Lundh B, Laurell A B. Studies of the third component of complement in synovial fluid from arthritic patients. II. Conversion and its relation to total complement. Clin Exp Immunol 1970; 6: 707-12.

3 Ruddy S, Fearon D T, Austen K F. Depressed synovial fluid levels of properdin and properdin factor B in patients with rheumatoid arthritis. Arthritis Rheum 1975; 18: 289-95.

4 Nydegger U E, Zubler R H, Gabay R, et al. Circulating complement breakdown products in patients with rheumatoid arthritis: correlation between plasma $\mathrm{C} 3 \mathrm{~d}$, circulating immune complexes, and clinical activity. $J$ Clin Invest 1977; 59: 862-8.

5 Perrin L H, Nydegger U E, Zubler R H, Lambert P H, Miescher $\mathrm{P}$ A. Correlation between levels of breakdown products of $\mathrm{C} 3, \mathrm{C} 4$ and properdin factor B in synovial fluids from patients with rheumatoid arthritis. Arthritis Rheum 1977; 20: $647-52$.

6 Hunder G G, McDuffie F C, Mullen B J. Activation of complement components $\mathrm{C} 3$ and factor $\mathrm{B}$ in synovial fluids. $J$ Lab Clin Med 1977; 89: 160-71.

7 Krauledat P B, Krapt F E, Manager B, Kalden J R. Evaluation of plasma $\mathrm{C} 3 \mathrm{~d}$ and immune complex determinations in the assessment of disease activity of patients with rheumatoid arthritis, systemic lupus erythematosus, and spondylitis. Rheumatol Int 1985; 5: 97-101.

8 Kerr M A. The human complement system: assembly of the classical pathway C3 convertase. Biochem J 1980; 189: 173-82.

9 Tanimoto K, Cooper N R, Johnson J S. Vaughan J H. Complement fixation by rheumatoid factor. J Clin Invest 1975; 55: $437-45$.

10 Schmid F R, Roitt I M, Rocha M J. Complement fixation by a two-component antibody system: immunoglobulin $G$ and immunoglobulin $\mathbf{M}$ anti-globulin (rheumatoid factor): paradoxical effect related to immunoglobulin $G$ concentration. $J$ Exp Med 1970; 132: 673-93.

11 Byers P H, Ward P A, Kellermeyer R W, Naff G B. Complement as a mediator of inflammation in acute gouty arthritis. II. Biological activities generated from complement by the interaction of serum complement and sodium urate crystals. J Lab Clin Med 1973; 81: 761-9.

12 Hasselbacher P. C3 activation by monosodium urate monohydrate and other crystalline material. Arthritis Rheum 1979; 22: $571-8$.

13 Doherty M, Whicher J T, Dieppe P A. Activation of the alternative pathway of complement by monosodium urate monohydrate crystals and other inflammatory particles. $A n n$ Rheum Dis 1983; 42: 285-91.

14 Tournes A S, Soura J M. Complement in synovial fluid. Johns Hopkins Medical Journal 1970; 127: 23-37.

15 Rynes R I, Ruddy S, Schur P H, Spragg J, Austen K F. Levels of complement components, properdin factors and kininogen in patients with inflammatory arthritides. J Rheumatol 1974; 1: 413-27.

16 Hasselbacher P. Immunoelectrophoretic assay for synovial fluid C3 with correction for synovial fluid globulin. Arthritis Rheum 1979; 22: 243-50.

17 Hunder G G, McDuffe F C, Clark R J. Consumption of C3 via the classical and alternative complement pathways by sera and synovial fluids from patients with rheumatoid arthritis. J Clin Lab Immunol 1979; 2: 269-73.

18 Kim H J, McCarty D J, Kozin F, Koethe S. Clinical significance of synovial fluid total hemolytic complement activity. $J$ Rheumatol 1980; 7: 143-52.

19 El-Ghobarey A F, Whaley K. Alternative pathway complement activation in rheumatoid arthritis. $J$ Rheumatol 1980; 7: 453-60.

20 Ochi T, Yonemasu K, Ono K. Immunochemical quantitations of $\mathrm{CI}$ components of $\mathrm{Clq}$ and $\mathrm{C} 3$ in sera and synovial fluid of patients with bone and joint disease. Ann Rheum Dis 1980; 39: 235-40.

21 Sheppeard H, Lea D J, Ward D J. Synovial fluid total hemolytic complement activity in rheumatic diseases-a reappraisal. $J$ Rheumatol 1981; 8: 390-7.

22 Inman R D, Harpel P C. CI inactivator-CIs complexes in inflammatory joint disease. Clin Exp Immunol 1983; 53: 521-8.

23 Berkowicz A, Kappelgaard E, Petersen J, et al. Complement $\mathrm{C} 3 \mathrm{c}$ and $\mathrm{C} 3 \mathrm{~d}$ in plasma and synovial fluid in rheumatoid arthritis. Acta Pathol Microbiol Immunol Scand [C] 1983; 91: 397-402.

24 Moxley G, Ruddy S. Elevated C3 anaphylatoxin levels in synovial fluids from patients with rheumatoid arthritis. Arthritis Rheum 1985; 28: 1089-95.

25 Mollnes T E, Lea T, Mellbye O J, Pahle J, Grand O, Harboe $M$. Complement activation in rheumatoid arthritis evaluated by C3dg and the terminal complement complex. Arthritis Rheum 1986; 29: 715-21.

26 Mollnes T E, Paus A. Complement activation in synovial fluid and tissue from patients with juvenile rheumatoid arthritis. Arthritis Rheum 1986; 29: 1359-64.

27 Brandslund I, Siersted H C, Svehag S E, Teisner B. Doubledecker rocket immunoelectrophoresis for direct quantitation of complement $\mathrm{C} 3$ split products with $\mathrm{C} 3 \mathrm{~d}$ specificities in plasma. $J$ Immunol Methods 1981; 44: 63-71.

28 A committee of the American Rheumatism Association. Revision of diagnostic criteria of rheumatoid arthritis. Arthritis Rheum 1959; 2: 16-20.

29 Powell R J, Leyland A M, Pound J D, Bossingham D H. An improved assay for IgG rheumatoid factor: its value in the diagnosis of rheumatoid arthritis. J Rheumatol 1985; 12: 427-31.

30 Deverill I. Kinetic measurements of the immunoprecipitin reaction using the centrifugal analyser. In: Price $C P$, Spencer $\mathrm{K}$, eds. Centrifugal analysers in clinical chemistry. London: Praeger, 1980: 109-24.

31 Nürnberger W, Bhakdi S. Plasma C3d/C3 quotient as a parameter for in vivo complement activation. J Immunol Methods 1984; 74: 87-91.

32 Vaughan J H, Boyles T B, Favor C V. Serum complement in rheumatoid arthritis. Am J Med Sci 1951; 222: 186-92.

33 Ruddy S, Colten H R. Rheumatoid arthritis: biosynthesis of complement proteins by synovial tissues. $N$ Engl J Med 1974; 290: $1284-8$.

34 Petz L D, Powers R, Fries J R, Cooper N R, Holman H R. The in vivo metabolism of the third component of complement in systemic lupus erythematosus. Arthritis Rheum 1977; 20: 1304-13.

35 Sliwinski A J,' Zvaifler N J. Decreased synthesis of the third component of complement (C3) in hypocomplementemic systemic lupus erythematosus. Clin Exp Immunol 1972; 11: 21-9.

36 McCarty D J, Kozin F. An overview of cellular and molecular mechanisms in crystal-induced inflammation. Arthritis Rheum 1975; 18: 757-64.

37 Reyes P A, Gobaira M J, Curd J G, Vaughan J H. Association of rheumatoid factor with complement activation in rheumatoid arthritis and other diseases. Clin Exp Immunol 1983; 53: 391-6.

38 Doherty M, Dieppe P A. Crystal deposition disease in the elderly. Clin Rheum Dis 1986; 12: 97-116.

39 Dieppe $\mathrm{P}$ A, Doherty $M$. The role of particles in the pathogenesis of joint disease. Curr Top Pathol 1982: 199-233.

40 Hasselbacher P. Crystal-protein interactions in crystal-induced arthritis. Advances in Inflammation Research 1982; 4: 25-44. 